\title{
Der Kühlungseffekt der personalisierten Lüftung
}

\author{
Hayder Alsaad, Conrad Völker \\ Bauhaus-Universität Weimar, Professur Bauphysik \\ Coudraystrasse $11 \mathrm{~A}$ \\ 99423 Weimar, Deutschland \\ Email: hayder.alsaad@uni-weimar.de, conrad.voelker@uni-weimar.de
}

(eingereicht Juli 2020; akzeptiert Juli 2020)

\section{Copyright-Vermerk}

Copyright 2020 Ernst \& Sohn. Dieser Artikel kann für den persönlichen Gebrauch heruntergeladen werden. Andere Verwendungen bedürfen der vorherigen Zustimmung der Autoren und des Verlags Ernst \& Sohn. Der folgende Artikel erschien in der Bauphysik 42 (2020), Heft 5, 218-225, DOI: 10.1002/bapi.202000018

\section{Zusammenfassung}

Personalisierte Lüftung (PL) kann die thermische Behaglichkeit sowie die Qualität der eingeatmeten Atemluft verbessern, in dem jedem Arbeitsplatz Frischluft separat zugeführt wird. In diesem Beitrag wird die Wirkung der PL auf die thermische Behaglichkeit der Nutzer unter sommerlichen Randbedingungen untersucht. Hierfür wurden zwei Ansätze zur Bewertung des Kühlungseffekts der PL untersucht: basierend auf (1) der äquivalenten Temperatur und (2) dem thermischen Empfinden. Grundlage der Auswertung sind in einer Klimakammer gemessene sowie numerisch simulierte Daten. Vor der Durchführung der Simulationen wurde das numerische Modell zunächst anhand der gemessenen Daten validiert. Die Ergebnisse zeigen, dass der Ansatz basierend auf dem thermischen Empfinden zur Evaluierung des Kühlungseffekts der PL sinnvoller sein kann, da bei diesem die komplexen physiologischen Faktoren besser berücksichtigt werden.

\section{Abstract}

Personalized ventilation (PV) can improve thermal comfort and inhaled air quality by supplying air to each workstation separately. This study investigates the impact of PV on the thermal state of the users under summer boundary conditions. Two approaches to evaluating the cooling effect of PV were investigated, based on equivalent temperature and based on thermal sensation. Both approaches implemented measured and simulated values of the cooling effect of PV. Before conducting the simulations, the numerical model was first validated against measured data collected in a climate chamber equipped with a thermal manikin. Results indicated that the thermal sensation approach can be more suitable for evaluating the cooling effect of PV due to the complex physiological factors it considers. 


\section{Einleitung}

Die globale Erwärmung und die daraus resultierenden steigenden Temperaturen erhöhen den Bedarf an fortschrittlichen Kühlungs- und Lüftungssystemen. Um weiterhin ein komfortables Raumklima gewährleisten zu können, wird in der Literatur unter anderem die personalisierte Lüftung empfohlen [1-3]. Die Personalisierte Lüftung (PL) ist ein System, dass saubere, temperierte Luft jedem Nutzer separat zur Verfügung stellt (Abb. 1). Durch dieses dezentrale System wird das Raumklima inhomogen, sodass Lufttemperatur, -geschwindigkeit und Strömungsrichtung in jeder Zone des Raumes unterschiedlich sind. Die PL wird typischerweise in Verbindung mit einer Lüftungsanlage eingesetzt, um eine individuelle Kontrolle über die lokale thermische Umgebung zu ermöglichen, ohne die anderen Menschen im Raum zu beeinträchtigen [2]. Dies führt zu einem Vorteil gegenüber der GesamtvolumenBelüftung, da die PL die individuellen Präferenzen der Nutzer hinsichtlich Lufttemperatur und -geschwindigkeit berücksichtigt; diese Präferenzen werden unter anderem durch Unterschiede in Bezug auf Kleidung, Geschlecht, metabolischer Energieumsatz und Akklimatisierung hervorgerufen [2,4]. Somit kann die PL den thermischen Komfort in der Arbeitsumgebung verbessern [5], auch bei hoher Raumtemperatur und Luftfeuchtigkeit [6]. Zusätzlich kann die individuelle Kontrolle eine psychologische Auswirkung haben, welche die Zufriedenheit der Nutzer mit ihrer thermischen Umgebung erhöht [7]. Durch diese thermische Zufriedenheit wird die Arbeitsleistung des Nutzers verbessert [8]. Die PL wird typischerweise durch eine Systemsteuerung kontrolliert, die sich am Schreibtisch des Nutzers befindet. Diese kann jedoch so eingestellt werden, dass sie automatisch auf die Anwesenheit des Benutzers reagiert. Wenn der Schreibtisch nicht besetzt ist, schaltet das Steuerungssystem die PL aus oder stellt sie auf einen minimalen Volumenstrom ein, was den Energieverbrauch des Lüftungssystems reduziert [2].

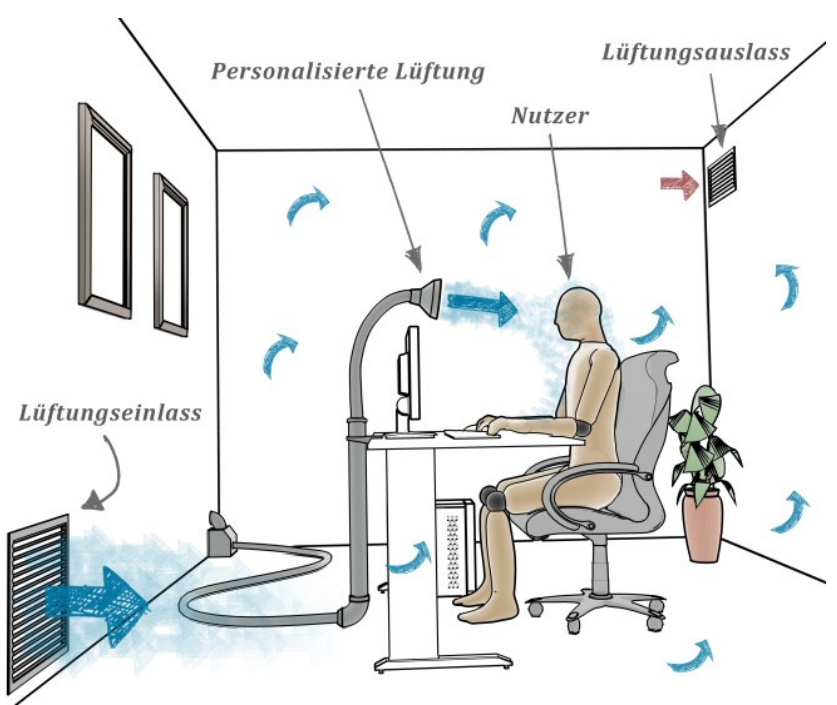

Abb. 1: Personalisierte Lüftung in einem Büroraum.

Fig. 1: Personalized ventilation in an office room.
Neben dem thermischen Komfort verbessert die PL auch die Luftqualität im Vergleich zu Gesamtvolumen-Systemen [10]. Um saubere Luft zur Einatmung bereitzustellen, muss die PL die konvektive Strömung, die den menschlichen Körper aufgrund seiner Wärmeabgabe umgibt, durchdringen (Abb. 2). Die für ein solches Eindringen erforderliche PL-Geschwindigkeit hängt von der Schichtdicke der konvektiven Strömung ab, die unter anderem von der Differenz zwischen der Lufttemperatur und der Oberflächentemperatur des $\mathrm{Nu}$ tzers abhängt $[2,11]$. Dieses Durchdringen ist bei wärmerer Raumtemperatur tendenziell einfacher, da die Dicke der konvektiven Grenzschicht dünner ist [11]

Der vorliegende Artikel fokussiert auf den Aspekt des thermischen Komforts bei der Verwendung der PL im Sommer. Zwei Methoden zur Bewertung des Kühlungseffekts wurden genutzt: (1) Äquivalente Temperatur sowie (2) thermisches Empfinden. Beide Methoden basieren auf Messungen in einer Klimakammer sowie numerischen Simulationen mit der CFD-Software ANSYS Fluent.

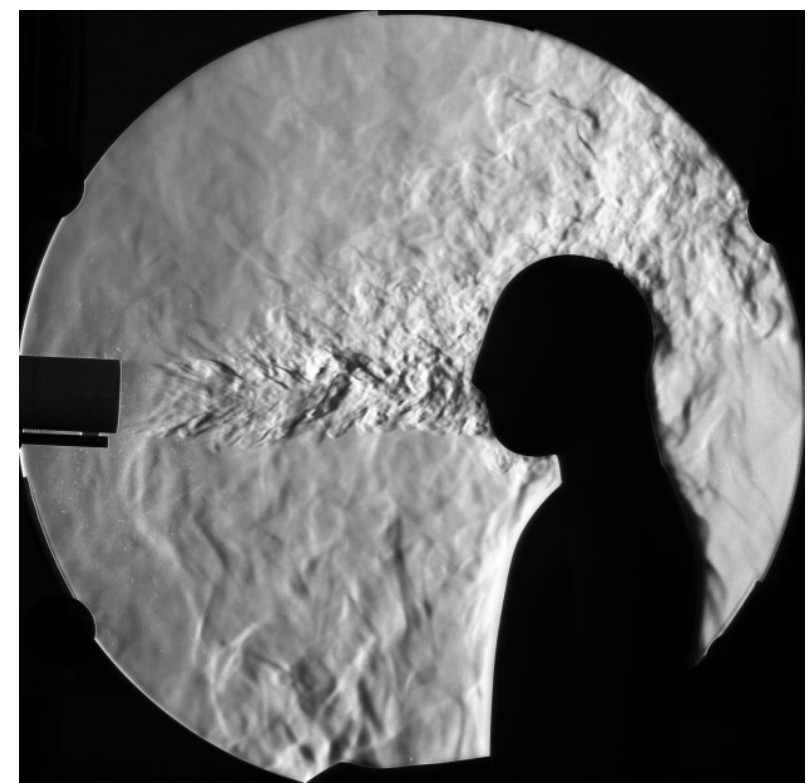

Abb. 2: Die Luftströmung einer PL (links), visualisiert mit dem Schlierenspiegel der Professur Bauphysik an der Bauhaus-Universität Weimar [9].

Fig. 2: The supplied flow from PV visualized using the schlieren mirror of the chair of building physics at the Bauhaus-Universität Weimar [9].

\section{Validierung des numerischen Modells}

Vor der Durchführung der eigentlichen Simulationen wurde das numerische Modell zuerst anhand gemessener Daten validiert. Die Messungen wurden in der Klimakammer der Professur Bauphysik an der Bauhaus-Universität Weimar durchgeführt. Die Klimakammer hat die Abmessungen 3 x 3 x 2,44 m (L x B x H) und wird mit Hilfe von wasserführenden Kapillarrohrmatten, die unter der Putzschicht integriert sind, temperiert. Die Temperatur jeder Oberfläche sowie des 

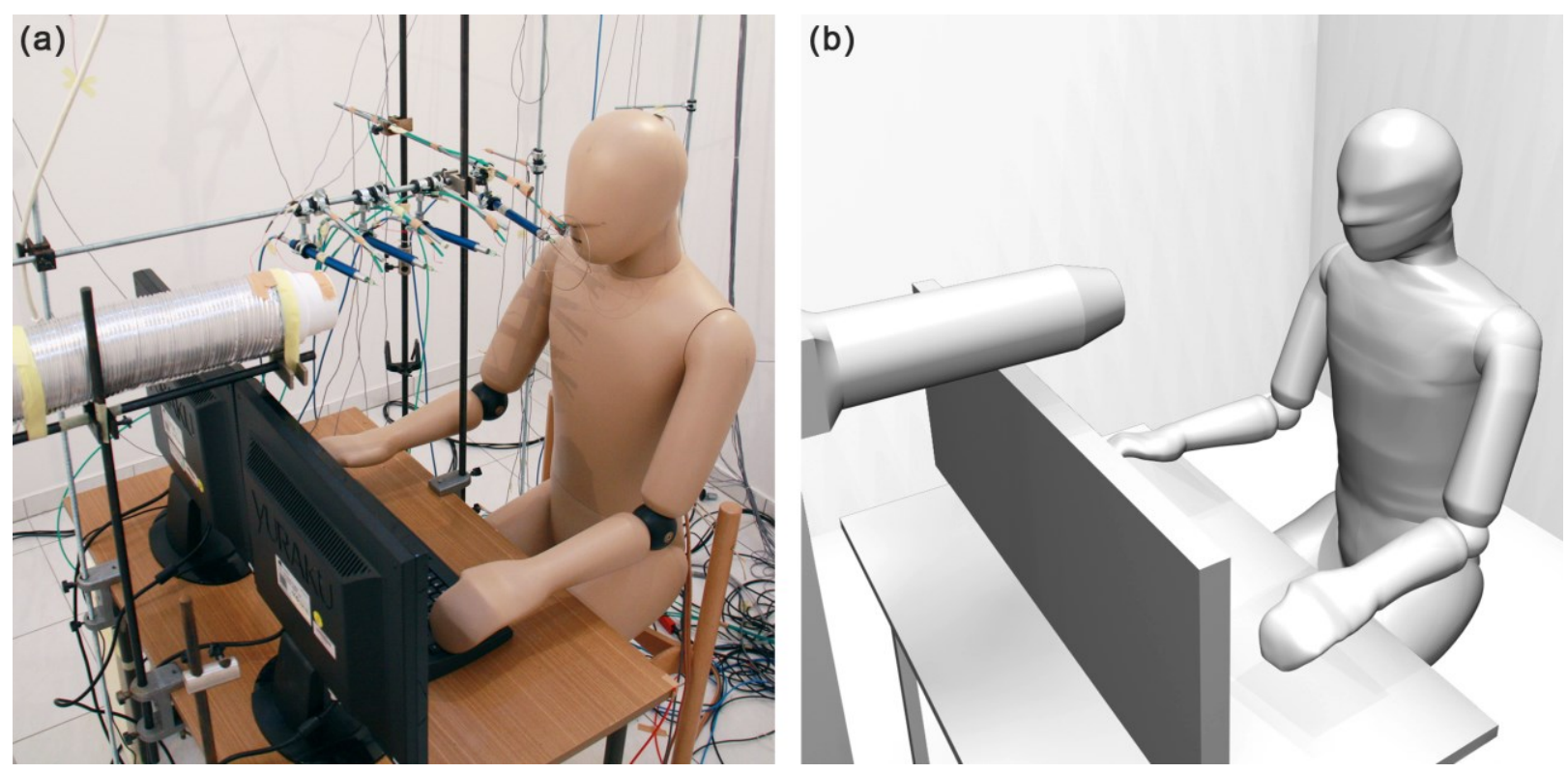

Abb. 3: (a) Der Messaufbau in der Klimakammer; (b) Die Geometrie der numerischen Modells.

Fig. 3: (a) The validation setup in the climate chamber; (b) the geometry of the numerical model.

Lüftungssystems kann separat angesteuert werden. Für die Messungen wurde eine übliche Bürokonfiguration bestehend aus einem Tisch, einem Computer und zwei Monitoren in der Klimakammer aufgebaut. Zusätzlich wurde ein thermisches Manikin („Feelix“) mit komplexer männlicher Körperform genutzt, um Geometrie und Wärmeabgabe des Nutzers zu simulieren. Dabei blieb das Manikin unbekleidet, ohne Perücke, ohne Atmung und saß auf einem offenen Stuhlgestell, um die Messungen und vor allem die nachfolgenden Simulationen zu vereinfachen (Abb. 3a). Gegenüber des Manikins wurde ein PLAuslass (Durchmesser 75mm) angebracht.

Die Validierung wurde bei zwei Raumtemperaturen durchgeführt $\left(\theta_{a \text {,room }}=26\right.$ und $\left.28^{\circ} \mathrm{C}\right)$. Die gesamte Raumlüftung (PL plus Raumlüftung) wurde auf $\dot{V}=32 \mathrm{~L} / \mathrm{s}$ gesetzt, wobei zwei Variationen durchgeführt wurden: $\dot{V}_{\text {chamber }}=28 \mathrm{~L} / \mathrm{s}$ mit $\dot{V}_{P L}=4 \mathrm{~L} / \mathrm{s}$ und $\dot{V}_{\text {chamber }}=27 \mathrm{~L} / \mathrm{s}$ mit $\dot{V}_{P L}=5 \mathrm{~L} / \mathrm{s}$. Diese beiden Einstellungen resultierten in einer Luftgeschwindigkeit am Gesicht von va,face $=0,36 \mathrm{~m} / \mathrm{s}$ beziehungsweise $0,66 \mathrm{~m} / \mathrm{s}$. Die Temperatur der Luft, die von dem PL-System bereitgestellt wurde, war um $3 \mathrm{~K}$ niedriger als die Raumlufttemperatur während der Fälle mit $\theta_{a \text {,room }}=26^{\circ} \mathrm{C}$ und $4 \mathrm{~K}$ während der Fälle mit $\theta_{a \text {,room }}=$ $28^{\circ} \mathrm{C}$. Zur Beurteilung der Luftqualität wurde Indikatorgas $\left(\mathrm{CO}_{2}\right)$ durch einen $\emptyset 4 \mathrm{~mm}$-Auslass an der Ecke des Schreibtisches in der Nähe des Manikins zugeführt ( $\left.\dot{V}_{\text {gas }}=3 \mathrm{~mL} / \mathrm{s}\right)$. Lufttemperatur, -geschwindigkeit und Indikatorgaskonzentrationen wurden an mehreren Punkten zwischen dem Gesicht des Manikins und dem PL-Auslass gemessen, um diese Werte mit dem numerischen Modell abzugleichen. Darüber hinaus wurde die Lufttemperatur und die Strömungsgeschwindigkeit an Mund/Nase unter verschiedenen Randbedingungen gemessen und simuliert.

Für die Validierung wurde die Geometrie des Messaufbaus in der Klimakammer sorgfältig vermessen und anschließend in ANSYS Workbench modelliert. Nur das Manikin wurde aufgrund seiner Komplexität als 3D-Scan in das numerische Modell implementiert. Abbildung 4 zeigt ein Beispiel der Validierungsergebnisse, die eine gute Übereinstimmung zwischen den gemessenen und simulierten Daten zeigen. Das validierte Modell verwendet das realizable k- $\varepsilon$ Turbulenzmodell mit einem Enhanced-Wall-Treatment und Full-BuoyancyEffects. Auftrieb wurde unter Verwendung des Incompressible-Ideal-Gas-Law simuliert. Zum Lösen der Gleichungen für Impuls, turbulenter kinetischer Energie und Dissipationsrate sowie Energie wurde das Coupled-Pressure-Velocity Scheme zusammen mit dem PRESTO-Spatial-Pressure DiscretizationScheme und dem Second-Order-Upwind DiscretizationScheme verwendet. Der Wärmeaustausch über Strahlung wurde nicht simuliert, da dem Großteil der Oberflächen eine Temperaturrandbedingung zugewiesen wurde. Eine vollständige Beschreibung der gelungenen Validierung kann in [12] gefunden werden.

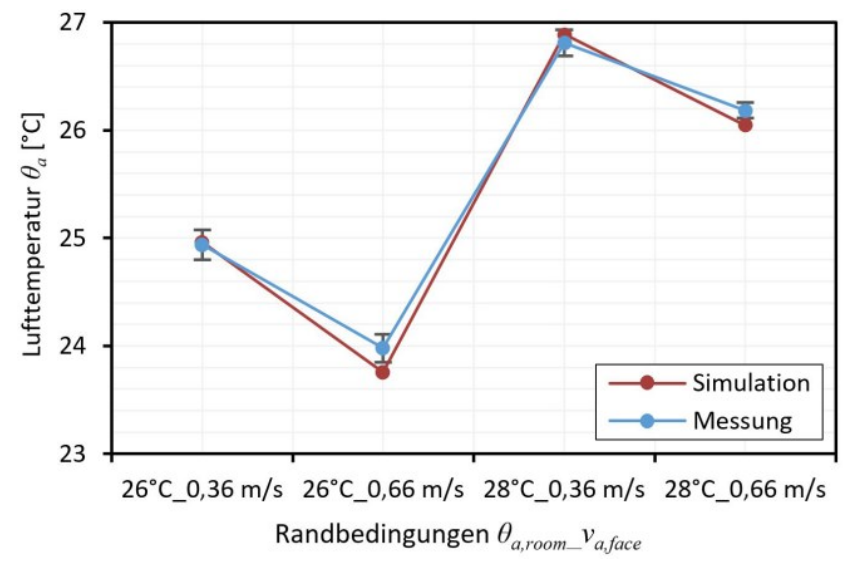

Abb. 4: Die gemessene und simulierte Lufttemperatur am Mund/Nase unter verschiedenen Randbedingungen.

Fig. 4: The measured and simulated inhaled air temperature under different boundary conditions. 


\section{Kühlungseffekt basierend auf äquiva- lenter Temperatur}

\subsection{Ermittlung}

Die in Kapitel 2 beschriebenen Aufbauten und Vorgehensweisen wurden eingesetzt, um den Kühlungseffekt der PL basierend auf der äquivalenten Temperatur zu simulieren. Zusätzlich wurden die in der Klimakammer gemessenen Daten verwendet, um den Kühlungseffekt zu berechnen, welcher wiederum mit den Simulationsergebnissen verglichen wurde.

Die äquivalente Temperatur, in die die gemessene Oberflächentemperatur sowie der Wärmestrom des thermischen Manikins einfließt, ist eine übliche Methode, die zur Berechnung des Kühlungseffekts einer PL unter Berücksichtigung des sensiblen Wärmeverlusts des Körpers eingesetzt werden kann [13]. Die äquivalente Temperatur ist definiert als die gleichmäßige Temperatur eines imaginären Gehäuses mit einer Luftgeschwindigkeit von null, bei der eine Person dieselbe sensible (trockene) Wärme über Strahlung und Konvektion (und ggf. auch Leitung) abgibt wie in der zu vergleichenden Umgebung [14]. Sie wird durch die folgende Gleichung berechnet:

$$
\theta_{e q, i}=\theta_{\text {skin, } i}-\frac{q_{i}}{h_{c a l i, i}}
$$

wobei $\theta_{\text {eq,i, }} \theta_{\text {skin,i, }} q_{i}$, und $h_{\text {cali,i }}$ jeweils die äquivalente Temperatur $\left[{ }^{\circ} \mathrm{C}\right]$, Oberflächentemperatur $\left[{ }^{\circ} \mathrm{C}\right]$, trockener Wärmeverlust $\left[\mathrm{W} / \mathrm{m}^{2}\right]$ und der Kalibrierungswärmeübergangskoeffizient $\left.\left[\mathrm{W} / \mathrm{m}^{2} \mathrm{~K}\right)\right]$ des Körperteils $i$ sind. Die globale äquivalente Temperatur wurde mittels flächengewichteter Mittelwerte von $\theta_{s \text { - }}$ kin, $q$, und $h_{\text {cali }}$ berechnet. Die äquivalente Temperatur (sowohl segmental als auch global) wird im vorliegenden Fall verwendet, um den Kühlungseffekt der inhomogenen thermischen Umgebung zu quantifizieren, in dem der Vergleich zu einem Referenzfall ohne PL gemäß der folgenden Gleichung gezogen wird:

$$
\Delta \theta_{e q, i}=\theta_{e q, i, P L}-\theta_{e q, i, r e f}
$$

wobei $\Delta \theta_{e q, i}$ der Kühlungseffekt der PL $\left[{ }^{\circ} \mathrm{C}\right], \theta_{e q, i, P L}$ die äquivalente Temperatur bei der Benutzung des PL-Systems [ $\left.{ }^{\circ} \mathrm{C}\right]$ und $\theta_{\text {eq,iref }}$ die äquivalente Temperatur während der Referenzfälle ohne das PL-System $\left[{ }^{\circ} \mathrm{C}\right]$ ist. Für diese Referenzfälle wurden weitere Messungen und Simulationen mit $\dot{V}_{\text {chamber }}=32 \mathrm{~L} / \mathrm{s}$ und $\dot{V}_{P L}=0 \mathrm{~L} / \mathrm{s}$ durchgeführt.

\subsection{Ergebnisse $\Delta \theta_{\text {eq, } i}$}

Die Simulationen zeigen, dass die PL einen deutlichen Kühlungseffekt erzeugt (Abb. 5). Dieser Effekt ist hauptsächlich lokal am Kopf (dem Ziel der zugeführten Strömung) spürbar, erwartungsgemäß erreicht dieser während der hohen PLStrömungsrate ( $\left.v_{a, f a c e}=0,66 \mathrm{~m} / \mathrm{s}\right)$ mit $\Delta \theta_{e q, i}=3,8 \mathrm{~K}$ das Maximum. Da ein Teil der Strömung den Brustbereich erreicht, wurde auch hier ein Kühlungseffekt von bis zu -1,4 K erzielt. Andere Körperteile wiederum werden von der PL nicht angeströmt, weshalb die PL einen geringeren Einfluss auf diese Segmente $\left(\Delta \theta_{e q, i}<0,5 \mathrm{~K}\right)$ hat. Da die gesamte äquivalente Temperatur unter Verwendung von flächengewichteten Mittelwerten von $\theta_{\text {skin, }} q$, und $h_{\text {cali }}$ berechnet wurde, war der gesamte Kühlungseffekt für den ganzen Körper mit einem Maximum von $0,6 \mathrm{~K}$ relativ gering.

Beim Vergleich des simulierten mit dem gemessenen Kühlungseffekt zeigen sich kleine Unterschiede. Bei geringeren Strömungsgeschwindigkeiten ( $v_{a}$,face $=0,36 \mathrm{~m} / \mathrm{s}$ ) gibt es eine Übereinstimmung mit einer maximalen Abweichung von 0,64 K. Andererseits resultieren die Fälle mit höheren Strömungsgeschwindigkeiten ( $v_{a}$ face $=0,66 \mathrm{~m} / \mathrm{s}$ ) in einer etwas größeren Abweichung (maximal 0,71 K zwischen Simulation und Messung).

Neben den möglichen numerischen Fehlern können die Unterschiede zwischen dem gemessenen und dem simulierten Kühlungseffekt auf Unterschiede in der gemessenen und simulierten Wärmestromdichte jedes Körpersegments zurückgeführt werden. Dem können zwei Ursachen zugrunde liegen: Obwohl ein 3D-Laserscanner verwendet wurde, um die
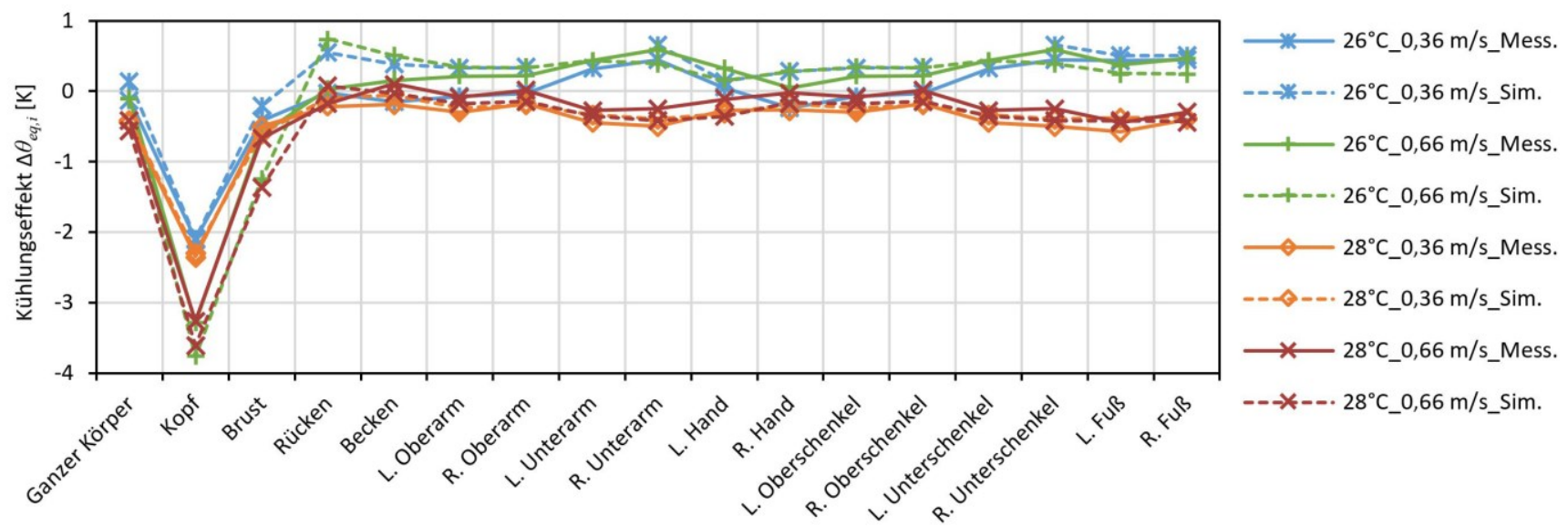

Abb. 5: Gemessener und simulierter Kühlungseffekt $\Delta \theta_{\text {eq,i }}$ einer $P L$.

Fig. 5: The measured and simulated cooling effect $\Delta \theta_{\text {eq,i }}$ when $P V$ was used. 
genaue Geometrie des thermischen Manikins zu erfassen, ist die Geometrie des gescannten Modells nicht komplett identisch mit der Geometrie des Manikins (Abb. 6). Außerdem wurde das Manikin aufgehangen, um in sitzender Position 3Dgescannt zu werden. Dieser Ansatz wurde verwendet, um das Manikin ohne Stuhl scannen zu können. Da das Manikin während der nun durchgeführten experimentellen Studie auf dem offenen Stuhl saß, ist es folglich schwierig, die genaue Haltung, die während des 3D-Scannens verwendet wurde, wiederherzustellen. Dies kann zu (kleinen) Unterschieden zwischen den experimentell ermittelten Wärmeübergangskoeffizient jedes Körperteils von denen der Simulation führen. Dies führt zu Unterschieden zwischen der gemessenen und der simulierten Wärmestromdichte, was zu einem Unterschied in der äquivalenten Temperatur gemäß Gleichung (1) führt.

Der zweite Grund für den Unterschied zwischen der gemessenen und der simulierten Wärmestromdichte sind unterschiedliche Ansätze bezüglich des Wärmetransports. Während der Messungen setzte sich die gesamte Wärmeabgabe des Manikins aus einem konvektiven und einem radiativen Anteil zusammen. Darüber hinaus war der Wärmeverlust durch Wärmeleitung vernachlässigbar, da das Manikin auf einem offenen Stuhlgestell saß und die Füße den Boden nicht berührten. Somit kann die gesamte Wärmestromdichte durch diese Gleichung ausgedrückt werden:

$$
q_{t}=q_{c}+q_{r}
$$

mit

$$
\begin{aligned}
& q_{c}=h_{c}\left(T_{s k i n}-T_{a}\right) \\
& q_{r}=\varepsilon \cdot \sigma \cdot\left(T_{2}^{4}-T_{1}^{4}\right)
\end{aligned}
$$
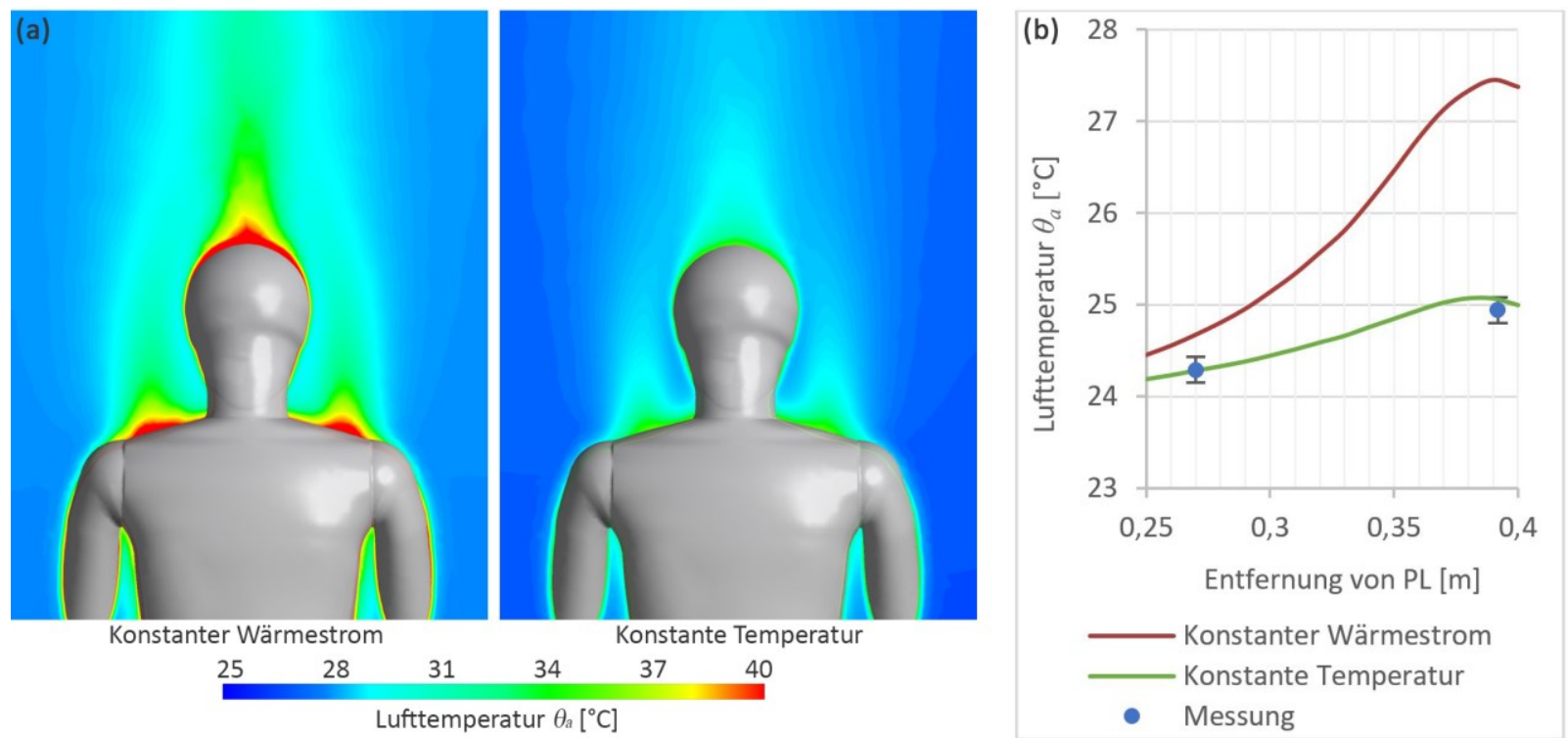

Abb. 7: Ein Vergleich zwischen CFD-Simulationen mit den Randbedingungen Wärmestromdichte und Temperatur; (a) das Mikroklima; (b) gemessene und simulierte Werte.

Fig. 7: A comparison between the fixed heat flux and fixed temperature boundary condition; (a) the microclimate; (b) measured and simulated values. 
Die Einschränkung des CFD-Modells, die entsprechenden Anteile der Wärmeabgabe (konvektiv, radiativ und evaporativ) genau zu simulieren ist der Grund, warum der Körper des Manikins als Temperatur-Randbedingung anstelle einer Wärmestromdichte definiert wurde. Abb. 7 zeigt die Ergebnisse einer Testsimulation, die den Einfluss der beiden unterschiedlichen Randbedingungen auf die konvektive Grenzschicht sowie den Plume zeigt. Abb. 7 (a) zeigt an, dass eine Wärmestromdichte-Randbedingung an einigen Körperteilen zu unrealistisch hohen Temperaturen führt, was mit den in [15] geschilderten Ergebnissen übereinstimmt. Diese Erhöhung führt zu einem größeren Temperaturgradienten zur umgebenden Raumluft, was wiederum höhere Dichtegradienten zur Folge hat. Resultat ist eine konvektive Grenzschicht mit höherer Luftgeschwindigkeit um den sitzenden Körper [11]. Diese Änderungen der Eigenschaften der thermischen Grenzschicht beeinflussen ihre Interaktion mit der PL-Strömung wesentlich, sodass die Beschaffenheit der Strömung am Körper stark verändert wird.

Abb. 7 (b) vergleicht den gemessen Wert der Lufttemperatur in der Nähe des Gesichtes mit dem simulierten Wert unter Verwendung der beiden definierten Grenzbedingungen bei $\theta_{\text {aroom }}=26^{\circ} \mathrm{C}$. Es zeigt sich, dass die Lufttemperatur am Gesicht signifikant höher ist, wenn die Wärmestromdichte als Randbedingung zugewiesen wird. In diesem Fall ist der konvektive Plume stärker ausgeprägt und hat eine höhere Luftgeschwindigkeit. Infolgedessen konnte in diesen Simulationen die Strömung der PL die Grenzschicht nicht durchdringen, um kühle Luft dem Gesicht zuzuführen. Daher wird empfohlen, bei solchen Simulationen jedem Segment der Geometrie des Manikins die Randbedingung Oberflächentemperatur zuzuweisen.

\section{Kühlungseffekt basierend auf äquiva- lenter Temperatur}

\subsection{Ermittlung}

Obwohl die äquivalente Temperatur eine übliche Methode für die Bewertung der thermischen Umgebung ist, fehlt ihr die Fähigkeit, den thermischen Zustand des Körpers umfänglich zu beschreiben. Zhang et al. [16] führt dies auf zwei Faktoren zurück: (1) Unterschiedliche Körperteile sind unterschiedlich empfindlich auf Veränderungen in der thermischen Umgebung, was je nach Körperteil zu verschiedenen physiologischen und psychologischen Auswirkungen führt. Da sowohl Messungen als auch Simulationen auf thermischen Manikins basieren und diese Unterschiede bezüglich der Körperteile berücksichtigt werden, reicht die äquivalente Temperatur nicht aus, um dieses Problem zu adressieren; (2) der Wärmeverlust durch Verdunstung des menschlichen Schweiß wird in der auf dem Manikin basierenden äquivalenten Temperatur normalerweise nicht in Betracht gezogen. Somit wird ein erhöhter Wärmeverlust durch gestiegene Luftgeschwindigkeiten nicht berücksichtigt, was folglich $\mathrm{zu}$ einer Unterschätzung des Kühlungseffekts des personalisierten Systems führt. Zusätzlich, wie in Abschnitt 3.2 erwähnt, kann die äquivalente Temperatur nicht den Kühlungseffekt des gesamten Körpers erfassen. Daher wurde Zhangs Modell [17] für thermisches Empfinden verwendet, um die Kühlungswirkung der PL zu beschreiben. Dieses Modell bewertet das lokale Empfinden basierend auf der Abweichung der Hauttemperatur von dem Sollwert, bei dem der Körper einen neutralen thermischen Zustand erfährt. Das lokale Empfinden wird als Funktion einer lokalen Hauttemperatur, der mittleren Hauttemperatur und der Kerntemperatur sowie der zeitlichen Änderung berechnet [18]:

$$
S_{i}=f\left(\theta_{\text {skin }, i}, \frac{d \theta_{\text {skin, }, i}}{d \theta}, \bar{\theta}_{\text {skin }}, \frac{d \theta_{\text {core }}}{d \theta}\right)
$$

Hierbei ist $S_{i}$ das lokale Empfinden des Körperteils $i, \theta_{s k i n, i}$ die lokale Hauttemperatur $\left[{ }^{\circ} \mathrm{C}\right], \bar{\theta}_{\text {skin }}$ die mittlere Hauttemperatur $\left[{ }^{\circ} \mathrm{C}\right]$ und $\theta_{\text {core }}$ die Körperkerntemperatur $\left[{ }^{\circ} \mathrm{C}\right]$. Das Empfinden wird auf einer 9-Punkte Skala ausgedrückt, wobei $-4=$ sehr kalt, $4=$ sehr heiß und $0=$ neutral ist. Ähnlich zur äquivalenten Temperatur wurde der empfindungsbasierte Kühlungseffekt als Differenz des thermischen Empfindens bei der Verwendung der PL und dem Referenzfall ohne PL bewertet:

$$
\Delta S_{i}=S_{i, P V}-S_{i, r e f}
$$

wobei $\Delta S_{i}$ der Kühlungseffekt des PL-Systems, $S_{i, P L}$ das Empfinden mit PL und $S_{i, r e f}$ das Empfinden während der Referenzfälle ohne PL ist.

Die Ermittlung des Empfindens wurde unter denselben im vorherigen Abschnitt beschriebenen Randbedingungen durchgeführt. Das simulierte Empfinden wurde durch die Kopplung von Strömungssimulation mit dem Komfortmodell der University of California, Berkeley (UCB) simuliert, welches sowohl Zhangs Empfindungsmodell als auch ein komplexes Thermoregulationsmodell umfasst. Dieses Modell erfasst komplexe physiologische Mechanismen wie Vasodilatation, Vasokonstriktion, Schwitzen und Stoffwechselrate [19]. Die Kopplung wurde erreicht durch das Importieren von Lufttemperatur und -geschwindigkeit um jedes Körpersegment in das UCBModell, damit die lokale Hauttemperatur mittels des Thermoregulationsmodells und anschließend das lokale und gesamte Empfinden mittels des Zhang-Modells berechnet wird. Neben den Simulationsergebnissen wurde, basierend auf der Oberflächentemperatur des Manikins in der Klimakammer, das thermische Empfinden manuell berechnet. Der simulierte und gemessene Kühlungseffekt wurde anschließend verglichen.

\subsection{Ergebnisse $\Delta S_{i}$}

Ähnlich wie unter 3.2 ist $\Delta S_{i}$ am Zielkörperteil (dem Kopf) hoch. Der maximale Kühlungseffekt tritt in der Simula- 


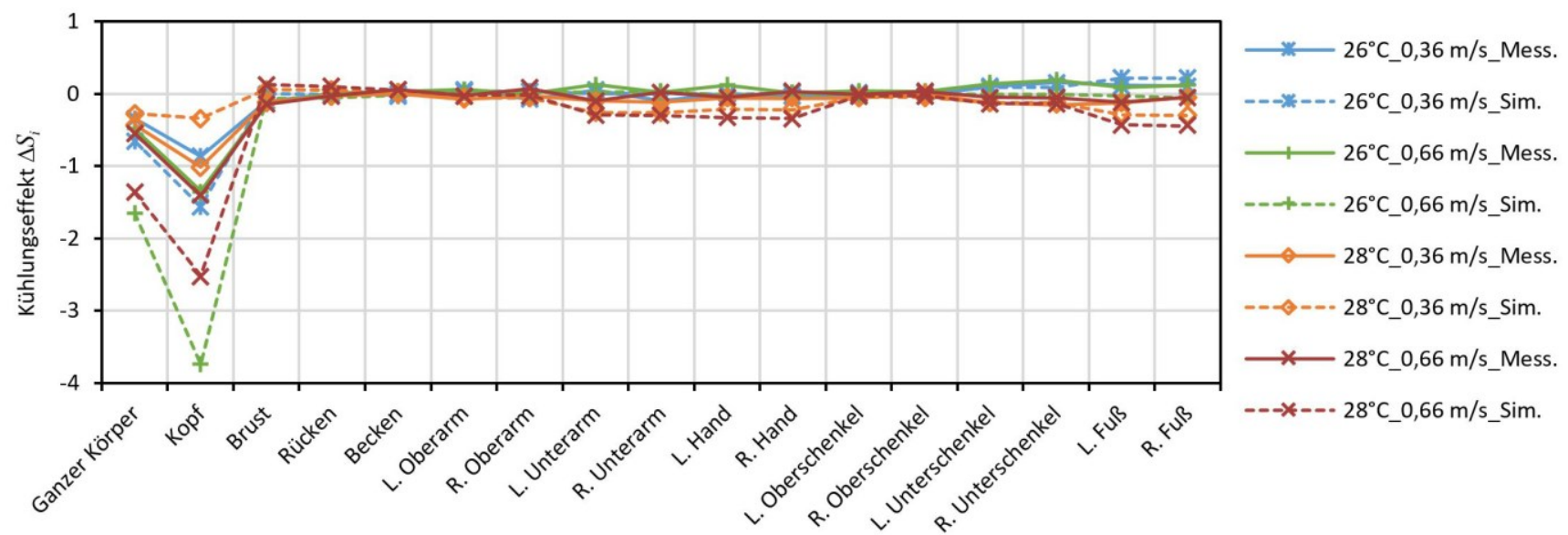

Abb. 8: Gemessener und simulierter Kühlungseffekt $\triangle S_{i}$ bei Nutzung der PL.

Fig. 8: Measured and simulated cooling effect $\Delta S_{i}$ when $P V$ was used.

tion bei hoher PL-Strömung va,face $=0,66 \mathrm{~m} / \mathrm{s}$ auf (Abb. 8). Interessanterweise zeigt das Brustkörpersegment, anders als $\Delta \theta_{e q, i}$, keinen wirklichen Kühlungseffekt $\left(\Delta S_{i}=-0,12\right)$. Der simulierte Kühlungseffekt des gesamten Körpers erreicht ein Maximum von $\Delta S_{i}=-1,7$.

Beim Vergleichen der simulierten und gemessenen Ergebnisse zeigen die Körperteile, die keiner direkten PLStrömung ausgesetzt waren (wie Oberschenkel, Beine, usw.), eine gute Übereinstimmung zwischen gemessenem und simuliertem PL-Kühlungseffekt. Nur wärmeempfindliche Körperteile (Füße und Hände) zeigen leichte Abweichungen. Andererseits stimmt der simulierte Kühlungseffekt am Kopf, der das von der PL-Strömung eigentlich betroffene Segment ist, mit den berechneten Werten nicht überein. Folglich ist auch der simulierte Kühlungseffekt des gesamten Körpers nicht mit den gemessenen Ergebnissen identisch. Die Unterschiede fallen in den Fällen $v_{a \text { aface }}=0,36 \mathrm{~m} / \mathrm{s}$ relativ gering aus. In diesen Fällen war die maximale Abweichungzwischen dem gemessenen und simulierten Kühlungseffekt $\Delta S_{i}=0,7$ am Kopf. Wenn die PLStrömung auf $v_{a \text {,face }}=0,66 \mathrm{~m} / \mathrm{s}$ erhöht wird, steigt die Abweichung signifikant auf 2,4 am Kopf.

Die Abweichung von den berechneten Ergebnissen wird durch mehrere Faktoren verursacht. Als erstes sind numerische Ungenauigkeiten sowohl beim UCB-Modell als auch beim Strömungssimulations-Modell ein Grund für einen Teil der Abweichung. Ein anderer wichtiger Grund für die Unterschiede ist, wie in Abschnitt 4 erwähnt, dass das thermische Manikin nur den trockenen Wärmeverlust abbildet. Im Gegenteil dazu berücksichtigt das UCB-Modell auch die menschliche Wärmeabgabe über die Verdunstung und Atmung.

\section{Zusammenfassung und Diskussion}

Es wurden zwei Ansätze zur Bewertung des Kühlungseffekts einer PL untersucht, basierend auf der äquivalenten Temperatur sowie dem thermischen Empfinden. Aus dem Vergleich der Ergebnisse der Abschnitte 3 und 4 lässt sich schlussfolgern, dass beide Ansätze nicht miteinander verglichen werden können. Der Ansatz des thermischen Empfindens zur Be- wertung des Kühlungseffekts liefert ein Ergebnis auf einer vordefinierten Skala, auf welcher jeder Wert einen bestimmten thermischen Zustand beschreibt. Das ergibt ein klares Verständnis für den Einfluss der PL auf das thermische Empfinden des Nutzers. Im Gegensatz dazu liefert der Ansatz der äquivalenten Temperatur einen Kühlungseffekt in Kelvin. Ein anderer Vorteil des thermischen Empfindens ist, dass der Unterschied in der thermischen Reaktion zwischen verschiedenen Körpersegment berücksichtigt wird. Darüber hinaus berücksichtigt es zahlreiche physiologische Faktoren, wie z.B. Schwitzen oder Atmung. Das ermöglicht dem Modell, den Effekt einer PL deutlich genauer zu berechnen, da die aus der PL resultierenden höheren Luftgeschwindigkeiten die Wärmeabgabe über Verdunstung fördern. Der Nachteil dieses Ansatzes ist, dass mit der vorhandenen Messtechnik keine Übereinstimmung zwischen den Messungen und Simulationen erzielt werden kann, obwohl sowohl das UCB-Modell als auch das Strömungssimulations-Modell separat anhand gemessener Daten validiert wurden. Grund hierfür ist die Komplexität des Thermoregulations-Modells im Vergleich zum verfügbaren thermischen Manikin. Andererseits ist durch die äquivalente Temperatur eine Übereinstimmung zwischen den Messungen und Simulationen relativ leicht zu erreichen, wobei allerdings der Kühlungseffekt der PL unterschätzt wird. Wenn die oben aufgelisteten Faktoren berücksichtigt werden, ist der Ansatz des thermischen Empfindens geeigneter, um den Einfluss der PL auf den thermischen Zustand besser zu untersuchen.

\section{Danksagung}

Wir möchten uns bei dem Deutschen Akademischen Austauschdienst (DAAD) für die Förderung dieses Forschungsprojekts (Programmnr.: 57129429) bedanken. Dank gilt auch Nele Ziegler für ihre Unterstützung beim Schreiben dieses Artikels.

\section{Literatur}

[1] Fanger, P. (2001) Human requirements in future air-conditioned environments in: International Journal of Refrigeration 24, H. 2, S. 148-153. 
[2] Melikov, A. (2004) Personalized ventilation in: Indoor Air 14, H. s7, S. 157-167.

[3] Melikov, A. (2016) Advanced air distribution: improving health and comfort while reducing energy use in: Indoor Air 26, H. 1, S. 112-124.

[4] Melikov, A. (2011) Advanced air distribution in: ASHRAE Journal 53, H. 11, S. 73-77.

[5] Alsaad, H.; Voelker, C. (2020) Performance evaluation of ductless personalized ventilation in comparison with desk fans using numerical simulations in: Indoor Air 30, H. 4, S. 776-789.

[6] Melikov, A.; Skwarczynski, M.; Kaczmarczyk, J.; Zabecky, J. (2013) Use of personalized ventilation for improving health, comfort, and performance at high room temperature and humidity in: Indoor Air 23, H. 3, S. 250-263.

[7] Bauman, F.; Carter, T.; Baughman, A.; Arens, E. (1998) Field study of the impact of a desktop task/ambient conditioning system in office buildings in: ASHRAE Transactions 104, S. 1153-1171.

[8] Tanabe, S.; Haneda, M.; Nishihara, N. (2015) Workplace productivity and individual thermal satisfaction in: Building and Environment 91, S. 42-50.

[9] Alsaad, H.; Voelker, C. (2020) Qualitative evaluation of the flow supplied by personalized ventilation using schlieren imaging and thermography in: Building and Environment 167, S. 106450.

[10] Fanger, 0. (2006) What is IAQ? in: Indoor Air 16, H. 5, S. 328-334.

[11] Voelker, C.; Maempel, S., Kornadt, O. (2014) Measuring the human body's microclimate using a thermal manikin in: Indoor Air 24, H. 6, S. 567-579.

[12] Alsaad, H.; Voelker, C. (2018) Performance assessment of a ductless personalized ventilation system using a validated CFD model in: Journal of Building Performance Simulation 11, H. 6, S. 689-704.

[13] Tanabe, S.; Arens, E.; Bauman, F.; Zhang, H.; Madsen, T. (1994) Evaluating thermal environments by using a thermal manikin with controlled skin surface temperature in: ASHRAE Transactions 100, Part 1, S. 39-48.

[14] SAE (1993) Equivalent temperature. SAE International, SAE Information Report, J2234.

[15] Voelker, C.; Alsaad, H. (2018) Simulating the human body's microclimate using automatic coupling of CFD and an advanced thermoregulation model in: Indoor Air 28, $\mathrm{H}$. 3, S. 415-425.

[16] Zhang, H.; Arens, E.; Zhai, Y. (2015) A review of the corrective power of personal comfort systems in non-neutral ambient environments in: Building and Environment 91, S. 15-41.

[17] Zhang, H. (2003) Human thermal sensation and comfort in transient and non-uniformthermal environment [Dissertation]. Center for the Built Environment, University of California, Berkeley.

[18] Zhang, H.; Arens, E.; Huizenga, C.; Han, T. (2010) Thermal sensation and comfort models for non-uniform and transient environments: Part I: Local sensation of individual body parts in: Building and Environment 45, H. 2, S. 380388.

[19] Huizenga, C.; Zhang, H.; Arens, E. (2001) A model of human physiology and comfort for assessing complex thermal environments in: Building and Environment 36, H. 6, S. 691-699. 